\title{
SMOOTH SOLUTIONS AND SINGULARITY FORMATION FOR THE INHOMOGENEOUS NONLINEAR WAVE EQUATION*
}

\author{
GENG $\mathrm{CHEN}^{\dagger}$ AND ROBIN YOUNG ${ }^{\ddagger}$
}

\begin{abstract}
We study the nonlinear inhomogeneous wave equation in one space dimension: $v_{t t}-T(v, x)_{x x}=0$. By constructing some "decoupled" Riccati type equations for smooth solutions, we provide a singularity formation result without restrictions on the total variation of unknown, which generalize earlier singularity results of Lax and the first author. These results are applied to several one-dimensional hyperbolic models, such as compressible Euler flows with a general pressure law, elasticity in an inhomogeneous medium, transverse MHD flow, and compressible flow in a variable area duct.
\end{abstract}

Key words. Wave equation, conservation laws, shock formation, nonlinear elasticity, compressible Euler equations, MHD.

AMS subject classifications. 35L65, 35B65, 35B35.

1. Introduction. In this paper, we consider the initial value problems for the second-order quasilinear inhomogeneous wave equation in one space dimension,

$$
v_{t t}-T(v, x)_{x x}=0
$$

where $(x, t) \in \mathbf{R} \times \mathbf{R}^{+}, v(x, t) \in \mathbf{R}$, and $T(v, x)$ is a smooth function satisfying

$$
T_{v}>0, \quad T_{v v}<0 .
$$

These assumptions imply that the equation is hyperbolic and genuinely nonlinear, so that solutions exhibit wave-like behavior. Signals propagate in a forward and backward direction with local nonlinear wavespeeds

$$
c=\sqrt{T_{v}} \text { and }-c=-\sqrt{T_{v}},
$$

respectively. Equation (1.1) includes a wide variety of interesting physical systems, such as one-dimensional nonlinear elasticity in an inhomogeneous medium [5, 6. In the context of elasticity, $x$ is a material coordinate, $v$ is the strain and $T$ is the elastic stress.

It is well known that solutions of nonlinear hyperbolic equations generally form shock waves in finite time. Shocks form as a result of gradient blowup, which is a consequence of genuine nonlinearity. In this paper, we study smooth solutions, and we are particularly interested in the lifetime of such smooth solutions. We study the dynamical system which governs the growth of gradients, and use this to give estimates on the lifetimes of smooth solutions. This was carried out for the homogeneous nonlinear wave equation, obtained by taking $T=T(v)$, by Lax in [7], and extensions to larger systems (with restrictions on the data) were obtained by John $[5]$ and Liu [10.

* Parts of this paper are included in this author's Ph.D dissertation at the University of Massachusetts, Amherst.

$\dagger^{\dagger}$ Department of Mathematics, Pennsylvania State University, University Park, PA, 16802 (chen@math.psu.edu).

${ }^{\ddagger}$ Department of Mathematics and Statistics, University of Massachusetts, Amherst, MA 01003 (young@math.umass.edu). Supported in part by NSF Applied Mathematics Grant Number DMS0908190. 
It is convenient to write (1.1) as a system, by setting

$$
u(x, t)=\int v_{t} d x, \quad p(v, x)=-T,
$$

which yields the first-order system

$$
\begin{aligned}
v_{t}-u_{x} & =0, \\
u_{t}+p(v, x)_{x} & =0,
\end{aligned}
$$

with

$$
p_{v}<0, \quad p_{v v}>0 .
$$

We make the further assumption that $p=p(v, x)$ is a $C^{3}$ function, and that boundedness of derivatives of $p$ and the sign conditions (1.4) are uniform in $x$. In particular, $p_{x}$ and $p_{x x}$ are uniformly bounded in $x$.

For smooth solutions, equations (1.2), (1.3) model compressible inviscid flow for general pressure laws in material coordinates (the Euler equations in a Lagrangian frame). Here $p$ is the pressure, $v$ is the specific volume, and $u$ is the fluid velocity. A general pressure is described by $p=p(v, S)$, but the entropy $S=S(x)$ is stationary as long as the flow is smooth, by the Second Law of Thermodynamics. Moreover, smooth solutions of the $5 \times 5$ system of one-dimensional transverse flow in Magnetohydrodynamics (MHD), which models a fluid coupled to a transverse (magnetic) vector field, and Eulerian flow in a variable area duct can also be modeled by (1.2), (1.3).

For homogeneous isentropic flow, we have $p=p(v)$, and (1.2), (1.3) is known as the the $p$-system. This is a simplified $2 \times 2$ system which admits a coordinate system of Riemann invariants, and for which Lax proved that any nontrivial data will form a shock wave in finite time [7. F. John and T.-P. Liu extended Lax's results to general systems of conservation laws of the form

$$
u_{t}+f(u)_{x}=0,
$$

with $u=\left(u_{1}, \cdots, u_{n}\right) \in \mathbf{R}^{n}$, for $n \geqslant 1$ and $(x, t) \in \mathbf{R} \times \mathbf{R}^{+}$. John proved that gradients will blow up for small compactly supported data, and this was later generalized by Liu, provided the total variation of the data is small enough [5, 10. There are also some singularity results for multi-dimensional conservation laws, subject to a restrictive "null condition" [12, 13. In a recent paper [1, the first author generalizes the singularity formation results in [7] to the $3 \times 3$ compressible Euler equations with polytropic ideal gas.

In this paper, we give singularity formation results for (1.2), (1.3), or equivalently (1.1). In [5, 10, small solutions are expanded asymptotically along integral curves, and wave interactions are treated quadratically. Here we take a different point of view, considering three wave families, namely forward and backward waves, as in the $p$-system, coupled with stationary waves, which carry entropy variations and inhomogeneity. We are then able to treat all waves without regard to their wave strength.

Our first task is to define the rarefactive and compressive character $(R / C$ character) of the nonlinear (non-stationary) waves in smooth solutions. In a $2 \times 2$ system, which is diagonal when expressed in Riemann invariants, it is clear when a wave is rarefying or compressing. However, in larger systems, this distinction is not clear as 
waves of different families generally interact continuously and cannot be decoupled . The $R / C$ character is a quantitative measure of how much rarefaction or compression is in the solution at any point.

In stationary solutions (including those with contact discontinuities), which include no compression or rarefaction, the pressure $p$ and velocity $u$ are constant, by the Rankine-Hugoniot conditions. We therefore use changes in $p$ (or $u$ ) to define the $R / C$ character of the solution. In doing so, we take the directional derivative along the opposite characteristic, to minimize the effect of waves of the opposite family, see [1. We use the superscripts " and "" to denote the directional derivatives along backward and forward characteristics, respectively, so that

$$
{ }^{\prime}=\partial_{t}-c \partial_{x} \quad \text { and } \quad{ }^{\prime}=\partial_{t}+c \partial_{x},
$$

where $c=\sqrt{-p_{v}}$ is the (local) wavespeed in Lagrangian coordinates, c.f. [7].

DeFinition 1. If the solutions of (1.2), (1.3) are smooth in an open set $U$ of the $(x, t)$-plane and $A$ is a point in $U$, then we say the solution is forward (backward) rarefactive at $A$, if and only if $p^{\prime}<0\left(p^{\prime}<0\right)$; it is forward (backward) compressive at $A$, if and only if $p^{\prime}>0\left(p^{\prime}>0\right)$.

It is convenient to introduce the change of variables

$$
h(v, x) \equiv \int_{v}^{v^{*}} \sqrt{-p_{v}} d v, \quad \text { and } \quad \mu \equiv x,
$$

where $v^{*}$ is a constant or infinity. Then by calculating $p^{\prime}$ and $p^{\prime}$, we introduce equivalent variables $\alpha$ and $\beta$, defined by

$$
\begin{aligned}
& \alpha \equiv-\frac{p^{\prime}}{c^{2}}=u_{x}+h_{x}+\frac{p_{\mu}}{c}, \\
& \beta \equiv-\frac{p^{\prime}}{c^{2}}=u_{x}-h_{x}-\frac{p_{\mu}}{c} .
\end{aligned}
$$

Here $u \pm h$ are the Riemann invariants for the corresponding isentropic $p$-system. Thus $\alpha$ and $\beta$ are direct generalizations of the derivatives of the Riemann invariants. For smooth solutions, we derive Riccati type ODEs for $\alpha$ and $\beta$, which provide a framework for studying smooth solutions and gradient blowup.

THEOREM 1. In (1.2), (1.3), smooth solutions satisfy

$$
\begin{aligned}
\alpha^{\prime} & =-\frac{c}{2}\left(\frac{p_{\mu}}{c}\right)_{h}(3 \alpha+\beta)+\frac{c_{h}}{2}\left(\alpha \beta-\alpha^{2}\right), \\
\beta^{\prime} & =\frac{c}{2}\left(\frac{p_{\mu}}{c}\right)_{h}(\alpha+3 \beta)+\frac{c_{h}}{2}\left(\alpha \beta-\beta^{2}\right),
\end{aligned}
$$

with $c_{h}>0$.

Equations (1.8) and (1.9) can be decoupled by use of an integrating factor. Define

$$
\begin{aligned}
& y \equiv \sqrt{c} \alpha-I=\sqrt{c}(u+h)_{x}+\frac{p_{\mu}}{\sqrt{c}}-I, \quad \text { and } \\
& q \equiv \sqrt{c} \beta+I=\sqrt{c}(u-h)_{x}-\frac{p_{\mu}}{\sqrt{c}}+I,
\end{aligned}
$$

where

$$
I=I(h, \mu) \equiv \int_{h_{0}}^{h} \frac{1}{2} \sqrt{c}\left(\frac{p_{\mu}}{c}\right)_{h} d h
$$


and $h_{0}$ is a constant.

THEOREM 2. For smooth solutions of (1.2), (1.3), we have

$$
\begin{aligned}
& y^{\prime}=a_{0}+a_{1} y-a_{2} y^{2}, \\
& q^{\prime}=a_{0}-a_{1} q-a_{2} q^{2},
\end{aligned}
$$

where

$$
\begin{aligned}
a_{0} & \equiv-c I_{\mu}+\frac{1}{2} \sqrt{c}\left(\frac{p_{\mu}}{c}\right)_{h} p_{\mu}-c\left(\frac{p_{\mu}}{c}\right)_{h} I-\frac{c_{h}}{2 \sqrt{c}} I^{2}, \\
a_{1} & \equiv-(2 \sqrt{c} I)_{h}, \\
a_{2} & \equiv \frac{c_{h}}{2 \sqrt{c}}>0 .
\end{aligned}
$$

We note that these are not closed ODEs, since both the directional derivatives and the coefficients are dependent on the underlying solution of (1.2), (1.3). Nevertheless, as in 7], we are able to compare them to closed ODEs and derive bounds on the lifespan of smooth solutions. For convenience, we only consider smooth, i.e. $C^{\infty}$, initial data, although our results also apply to $C^{2}$ initial data.

THEOREM 3. Assume the smooth solution takes values in some compact set $\mathcal{K}$, uniformly in $x$. Then there is some constant $N>0$ depending only on $\mathcal{K}$, such that, if $y$ or $q$ is less than $-N$ somewhere in the initial data, then $\left|u_{x}\right|$ and/or $\left|v_{x}\right|$ blow up in finite time: that is, there is some critical time $T_{*}<\infty$, so that

$$
\max \left\{\left|u_{x}\right|,\left|v_{x}\right|\right\} \rightarrow \infty \quad \text { as } t \rightarrow T_{*} .
$$

Blowup of the gradient does not mean that the solution fails to exist: rather, this usually heralds the formation of a shock, and the associated decay of solutions. Generally, solutions are continued as weak solutions which contain shocks. However, in order to study shock propagation our system needs to be in conservation form, and we do not consider those issues here.

Our assumption that the solution stay in a compact set $\mathcal{K}$ can be stated technically as

$$
\begin{gathered}
|h|<B_{1}, \quad A_{2}<c<B_{2}, \quad A_{3}<c_{h}<B_{3}, \\
\left|c_{\mu}\right|<B_{4}, \quad\left|c_{\mu \mu}\right|<B_{5}, \quad\left|c_{h \mu}\right|<B_{6}, \quad\left|p_{\mu}\right|<B_{7}, \quad\left|p_{\mu \mu}\right|<B_{8},
\end{gathered}
$$

where $A_{i}$ and $B_{i}$ are all positive constants. These conditions simply mean that we do not leave the domain $\mathcal{K}$ in the solutions we consider, and allow us to stay away from vacuum and other points where the equation may become singular. From a practical point of view, the only restriction on the data (apart from smoothness conditions) is that the solution stays away from vacuum: in general we do not expect the pressure $p$ to grow unboundedly. For the $p$-system, an invariant region provides an upper bound for the pressure, and although there is not an invariant region for the full system, physically we expect the pressure to remain finite. We note that the assumption that the solution does not form a vacuum is implicit in [7, but this is reasonable as a vacuum cannot form in finite time, see [17, 18.

Theorem 3 implies that gradients of solutions blow up if the initial compressions are strong enough. When the variation of entropy is mild, $N$ is close to zero, so the 
shock free solutions are "almost rarefactive", which is consistent with Lax's singularity formation results in [7]. In [14] and a forthcoming paper [2, examples of solutions containing compressive waves are constructed, but the gradients of those solutions remain finite.

In a series of recent papers 14, 15, 16, the possibility of time-periodic solutions in the compressible Euler equations has been demonstrated. A critical feature of this study is how the $R / C$ structure of waves can change across a contact discontinuity. In this paper we check the consistency of our results, which presuppose a smooth entropy field, with those results. By studying the $R / C$ structure and the way in which it can change further, we expect eventually to see time-periodic solutions as in [14, 15] with piecewise smooth entropy, consisting of both contact discontinuities and smooth entropy variations.

Our results apply directly to a number of systems having structure similar to the inhomogeneous nonlinear wave equation. In addition to equations (1.2), (1.3), we apply these ideas to transverse flow in one-dimensional magnetohydrodynamics (MHD), a $5 \times 5$ system modeling fluid motion coupled to a transverse magnetic field. By restricting to a polytropic ideal gas, we are able to give a stronger singularity formation result than that of Theorem 3, similar to that of [1. Finally, we consider inviscid compressible flow in a varying area duct, to which similar ideas apply.

The paper is arranged as follows. In Section 2, we give the background of the equations and establish some useful identities. In Section 3, we define rarefactive and compressive waves. In Sections 4 and 5 , we prove Theorems 13 , respectively. In Section 6, we consider the consistency of $R / C$ structures, and in Sections 7 and 8 , we apply our ideas to one-dimensional transverse flow of MHD and compressible flow in a varying duct.

2. Coordinates and background. We focus on equations (1.2), (1.3) from now on. We assume that $p(v, x)$ is a smooth function of $v$ and $x$, satisfying

$$
p_{v}<0, \quad \text { and } p_{v v}>0,
$$

for all $x$ and $v=v(x, t) \in(0, \infty)$. These conditions imply hyperbolicity and genuine nonlinearity, respectively. The vacuum state corresponds to $v=\infty$; however, since we assume the data remains in a compact set, we will not address questions at vacuum. The local absolute wavespeed is

$$
c(v, x) \equiv \sqrt{-p_{v}}>0 .
$$

We make the change of variables

$$
h(v, x) \equiv \int_{v}^{v^{*}} c d v=\int_{v}^{v^{*}} \sqrt{-p_{v}} d v,
$$

see (1.7), where $v^{*}>0$ is a convenient constant (or $\infty$ if the integral converges uniformly). Since $p(v, x)$ is smooth, the function $h(v, x)$ is also smooth with respect to $v$ and $x$. Moreover, since

$$
h_{v}(v, x)=-c<0,
$$

the inverse function $v=v(h, \mu)$ is smooth with respect to $h$ and $\mu$, where we have set

$$
\mu \equiv x
$$


For any function $f(v, x)$, we will write

$$
f(h, \mu)=f(h(v, x), \mu)=f(v, x),
$$

without ambiguity, and we use the subscript notation

$$
\begin{gathered}
f_{x}=\frac{\partial}{\partial x} f(v(x, t), x), \quad f_{\bar{x}}=\frac{\partial}{\partial x} f(v, x), \quad f_{v}=\frac{\partial}{\partial v} f(v, x), \\
f_{h}=\frac{\partial}{\partial h} f(h, \mu), \quad f_{\mu}=\frac{\partial}{\partial \mu} f(h, \mu),
\end{gathered}
$$

for the various partial derivatives of $f$.

We can relate the different partial derivatives as follows: by (2.2),

$$
v_{h}=-\frac{1}{c}, \quad \text { so that } \quad v_{t}=v_{h} h_{t}=-\frac{1}{c} h_{t} .
$$

Furthermore, since

$$
-c^{2}=p_{v}=p_{h} h_{v}=-c p_{h}, \quad \text { we have } \quad p_{h}=c,
$$

and so

$$
p_{x}=p_{h} h_{x}+p_{\mu}=c h_{x}+p_{\mu} .
$$

Thus, for smooth solutions, (1.2), (1.3) can be written as

$$
\begin{aligned}
h_{t}+c u_{x} & =0, \\
u_{t}+c h_{x}+p_{\mu} & =0 .
\end{aligned}
$$

Next, differentiating $v=v(h(v, x), \mu)$ with respect to $x$, and recalling (2.3) and (2.4), we get

$$
0=v_{h} h_{\bar{x}}+v_{\mu}, \quad \text { so that } \quad v_{\mu}=-v_{h} h_{\bar{x}}=\frac{h_{\bar{x}}}{c} .
$$

Similarly, for any function $f(v, x)$, we get

$$
\begin{aligned}
f_{\mu} & =f(v(h, \mu), x)_{\mu} \\
& =f_{v} v_{\mu}+f_{\bar{x}} \\
& =\frac{f_{v}}{c} h_{\bar{x}}+f_{\bar{x}},
\end{aligned}
$$

and

$$
f_{h}=f_{v} v_{h}=-\frac{f_{v}}{c} .
$$

It follows that, for smooth solutions, $p$ and $c$ are smooth with respect to $h$ and $\mu$. Moreover, using (2.8) and (2.9), any quantities such as the $R / C$ character, ODEs, and singularity formation results in this paper can all be expressed in the variables $(v, x)$ instead of $(h, \mu)$. 
3. Compressive and Rarefactive waves. In this section, we define the rarefactive $(R)$ and compressive $(C)$ characters of (1.2), (1.3), which quantitively indicate the amount of rarefaction or compression in the solutions at any point.

We first consider isentropic flow, for which $p=p(v)$ and our system reduces to the $p$-system,

$$
\begin{aligned}
& h_{t}+c u_{x}=0, \\
& u_{t}+c h_{x}=0 .
\end{aligned}
$$

The Riemann invariants $s=u+h$ and $r=u-h$ satisfy the diagonal system

$$
s_{t}+c s_{x}=0, \quad r_{t}-c r_{x}=0,
$$

and so are constant along forward and backward characteristics,

$$
\frac{d x}{d t}=+c, \quad \frac{d x}{d t}=-c
$$

respectively. In an isentropic domain, because the system is diagonal, it is clear when waves are compressive or rarefactive: indeed, the amount of compression or rarefaction can be measured by derivatives of the appropriate Riemann invariant. There are several equivalent conditions, and for us it is convenient to consider the change in pressure: if $p$ decreases as we traverse the wave from front to back, the wave is rarefactive $(R)$, while if $p$ increases, it is compressive $(C)$ [1, 7, 17. This is consistent with the entropy condition for shocks, which states that the pressure is always larger behind a shock.

When $p=p(v, x)$ explicitly depends on $x$, we first consider stationary solutions, in which there are no compressive or rarefactive waves, so the $R / C$ characters should vanish. In stationary solutions, the pressure $p$ is constant,

$$
p_{t}=p_{v} v_{t}=0 \quad \text { and } \quad p_{x}=-u_{t}=0
$$

so its directional directives are zero. Physically, in gas dynamics, this means that pressure is not impacted by the variation of entropy, c.f. [1]. Thus, by considering the directional derivatives of $p$ along the opposite characteristics, we obtain Definition 1 : the solution is forward (backward) rarefactive at $A$, if and only if $p^{\prime}<0\left(p^{\prime}<0\right)$; it is forward (backward) compressive at $A$, if and only if $p^{\prime}>0\left(p^{\prime}>0\right)$.

We could also use $u$ to define the $R / C$ character, as this is also constant in stationary solutions. We define

$$
\alpha \equiv-\frac{p^{\prime}}{c^{2}} \quad \text { and } \quad \beta \equiv-\frac{p^{\prime}}{c^{2}} .
$$

Lemma 1. For smooth solutions of (1.2), (1.3), we have

$$
p^{\prime}=-c u^{\prime} \quad \text { and } \quad p^{\prime}=c u^{\prime}
$$

while also

$$
\alpha=u_{x}+h_{x}+\frac{p_{\mu}}{c} \quad \text { and } \quad \beta=u_{x}-h_{x}-\frac{p_{\mu}}{c} .
$$


Proof. By (1.2), (1.3) and (2.1),

$$
c u^{\prime}=c u_{t}+c^{2} u_{x}=-c p_{x}-p_{v} v_{t}=-p^{\prime},
$$

and similarly $p^{\prime}=c u^{\prime}$. By (2.5) and (2.6),

$$
\begin{aligned}
-c^{2} \beta & =p^{\prime}=p_{t}+c p_{x} \\
& =p_{h} h_{t}+c\left(p_{h} h_{x}+p_{\mu}\right) \\
& =-c^{2}\left(u_{x}-h_{x}-\frac{p_{\mu}}{c}\right),
\end{aligned}
$$

and similarly for $\alpha$, so (3.3) follows.

COROLlary 1. The $R / C$ character of a smooth solution is given by:

$$
\begin{array}{llll}
\text { Forward } & R & \text { iff } & \alpha>0, \\
\text { Forward } & C & \text { iff } & \alpha<0, \\
\text { Backward } & R & \text { iff } & \beta>0, \\
\text { Backward } & C & \text { iff } & \beta<0 .
\end{array}
$$

Moreover, provided the solution values remain in $\mathcal{K}$,

$$
|\alpha| \text { or }|\beta| \rightarrow \infty \quad \text { iff }\left|u_{x}\right| \text { or }\left|v_{x}\right| \rightarrow \infty \text {. }
$$

Proof. Clearly, by (3.3),

$$
p^{\prime} \gtrless 0 \Leftrightarrow \alpha \lessgtr 0, \quad \text { and } \quad p^{\prime} \gtrless 0 \Leftrightarrow \beta \lessgtr 0 .
$$

By Lemma 1,

$$
\alpha+\beta=2 u_{x}, \quad \alpha-\beta=2\left(h_{x}+\frac{p_{\mu}}{c}\right),
$$

and (3.4) follows since $p_{\mu}$ and $c$ remain finite. $\square$

In an isentropic domain, i.e. $p=p(v)$, it is clear that

$$
s_{x}=\alpha \quad \text { and } \quad r_{x}=\beta,
$$

so we can regard $\alpha$ and $\beta$ as direct generalizations of the derivatives of the Riemann invariants.

4. Differential equations for gradients. In this section, we consider the characteristic decompositions of smooth solutions. By considering the directional derivatives of $\alpha$ and $\beta$, we derive the ODEs for $\alpha$ and $\beta$ as stated in Theorem 1

Proof of Theorem 1. We show (1.8), since (1.9) follows in exactly the same way. We have

$$
\begin{aligned}
\alpha^{\prime} & =\left(u_{x}+h_{x}+\frac{p_{\mu}}{c}\right)_{t}+c\left(u_{x}+h_{x}+\frac{p_{\mu}}{c}\right)_{x} \\
& =\left(u_{x t}+c h_{x x}\right)+\left(h_{x t}+c u_{x x}\right)+\left(\frac{p_{\mu}}{c}\right)_{t}+c\left(\frac{p_{\mu}}{c}\right)_{x} \\
& =\left(u_{t}+c h_{x}\right)_{x}+\left(h_{t}+c u_{x}\right)_{x}-c_{x} h_{x}-c_{x} u_{x}+\left(\frac{p_{\mu}}{c}\right)_{t}+c\left(\frac{p_{\mu}}{c}\right)_{x} .
\end{aligned}
$$

By (2.6), (2.7),

$$
\left(u_{t}+c h_{x}\right)_{x}=\left(-p_{\mu}\right)_{x}=-p_{\mu h} h_{x}-p_{\mu \mu},
$$


and

$$
\left(h_{t}+c u_{x}\right)_{x}=0 .
$$

Thus the right hand side of (4.1) is

$$
-p_{\mu h} h_{x}-p_{\mu \mu}-\left(c_{h} h_{x}+c_{\mu}\right)\left(h_{x}+u_{x}\right)+\left(\frac{p_{\mu}}{c}\right)_{h}\left(h_{t}+c h_{x}\right)+c\left(\frac{p_{\mu}}{c}\right)_{\mu},
$$

since $f_{x}=f_{h} h_{x}+f_{\mu}$ for any function $f$.

By (3.3) and (2.6), we have

$$
h_{x}=\alpha-u_{x}-\frac{p_{\mu}}{c}, \quad \text { and } \quad h_{t}=-c u_{x},
$$

and by (2.5),

$$
c\left(\frac{p_{\mu}}{c}\right)_{h}=c_{\mu}-c_{h} \frac{p_{\mu}}{c} .
$$

Thus (4.2) can be simplified to

$$
\begin{gathered}
-p_{\mu h}\left(\alpha-u_{x}-\frac{p_{\mu}}{c}\right)-p_{\mu \mu}-\left(c_{h} \alpha-c_{h} u_{x}-c_{h} \frac{p_{\mu}}{c}+c_{\mu}\right)\left(\alpha-\frac{p_{\mu}}{c}\right) \\
+\left(\frac{p_{\mu}}{c}\right)_{h}\left(c \alpha-2 c u_{x}-p_{\mu}\right)+c\left(\frac{p_{\mu}}{c}\right)_{\mu} \\
=-c\left(\frac{p_{\mu}}{c}\right)_{h} u_{x}+\left[c_{h} u_{x}-c\left(\frac{p_{\mu}}{c}\right)_{h}\right] \alpha-c_{h} \alpha^{2} .
\end{gathered}
$$

Finally, making the substitution

$$
u_{x}=\frac{\alpha+\beta}{2}
$$

yields (1.8).

By (1.4), (2.1) and (2.2),

$$
0>\left(\sqrt{-p_{v}}\right)_{v}=c_{v}=c_{h} h_{v}=c_{h}(-c),
$$

so $c_{h}>0$, and the theorem is proved.

Corollary 2. For smooth solutions of (1.2), (1.3), we have

$$
\alpha^{\prime}=-\frac{c}{4}\left(\frac{p_{\bar{x}}}{p_{v}}\right)_{v}(3 \alpha+\beta)-\frac{c_{v}}{2 c}\left(\alpha \beta-\alpha^{2}\right),
$$

and

$$
\beta^{\prime}=\frac{c}{4}\left(\frac{p_{\bar{x}}}{p_{v}}\right)_{v}(\alpha+3 \beta)-\frac{c_{v}}{2 c}\left(\alpha \beta-\beta^{2}\right) .
$$

Proof. By (2.1), (2.2), (2.8), (2.9) and (4.3), we have

$$
\begin{aligned}
c\left(\frac{p_{\mu}}{c}\right)_{h} & =c_{\mu}-c_{h} \frac{p_{\mu}}{c} \\
& =c_{\bar{x}}+\frac{c_{v}}{c^{2}} p_{\bar{x}} \\
& =\frac{c}{2}\left(\frac{p_{\bar{x}}}{p_{v}}\right)_{v},
\end{aligned}
$$


and by (4.4),

$$
\frac{c_{h}}{2}=-\frac{c_{v}}{2 c},
$$

and the corollary follows from Theorem 1, $\mathrm{u}$

We now make another change of variables and transform (1.8) and (1.9) into decoupled differential equations by use of an integrating factor.

Proof of Theorem Q First, the condition $a_{2}>0$ follows immediately from (4.4).

By (3.3) and (2.6),

$$
h^{\prime}=h_{t}+c h_{x}=-c u_{x}+c h_{x}=-c\left(\beta+\frac{p_{\mu}}{c}\right),
$$

so that

$$
\beta=-\frac{h^{\prime}}{c}-\frac{p_{\mu}}{c}
$$

Hence, (1.8) can be written

$$
\alpha^{\prime}=-\frac{c}{2}\left(\frac{p_{\mu}}{c}\right)_{h}\left(3 \alpha-\frac{h^{\prime}}{c}-\frac{p_{\mu}}{c}\right)+\frac{c_{h}}{2} \alpha\left(-\frac{h^{\prime}}{c}-\frac{p_{\mu}}{c}\right)-\frac{c_{h}}{2} \alpha^{2} .
$$

We move the terms including $h^{\prime}$ to the left hand side, so

$$
\begin{aligned}
\alpha^{\prime}-\frac{1}{2}\left(\frac{p_{\mu}}{c}\right)_{h} h^{\prime}+\frac{c_{h}}{2 c} \alpha h^{\prime} \\
=\frac{1}{2}\left(\frac{p_{\mu}}{c}\right)_{h} p_{\mu}+\left(-\frac{3}{2} c\left(\frac{p_{\mu}}{c}\right)_{h}-\frac{c_{h}}{2 c} p_{\mu}\right) \alpha-\frac{c_{h}}{2} \alpha^{2} .
\end{aligned}
$$

Now, by (2.5),

$$
-\frac{3}{2} c\left(\frac{p_{\mu}}{c}\right)_{h}-\frac{c_{h}}{2 c} p_{\mu}=-c\left(\frac{p_{\mu}}{c}\right)_{h}-\frac{p_{\mu h}}{2}=-c\left(\frac{p_{\mu}}{c}\right)_{h}-\frac{c_{\mu}}{2},
$$

and, since $\mu^{\prime}=c$, we have

$$
\sqrt{c} \alpha^{\prime}+\frac{c_{h}}{2 \sqrt{c}} \alpha h^{\prime}+\frac{\sqrt{c}}{2} c_{\mu} \alpha=(\sqrt{c} \alpha)^{\prime} .
$$

Thus, multiplying (4.6) by $\sqrt{c}$ and simplifying, we get

$$
\begin{aligned}
& (\sqrt{c} \alpha)^{\prime}-\frac{1}{2} \sqrt{c}\left(\frac{p_{\mu}}{c}\right)_{h} h^{\prime} \\
& \quad=\frac{1}{2} \sqrt{c}\left(\frac{p_{\mu}}{c}\right)_{h} p_{\mu}-c \sqrt{c}\left(\frac{p_{\mu}}{c}\right)_{h} \alpha-\frac{c_{h} \sqrt{c}}{2} \alpha^{2} .
\end{aligned}
$$

By (1.10),

$$
\alpha=\frac{y+I}{\sqrt{c}},
$$

where $I$ is defined in (1.12) and satisfies

$$
I^{\prime}=I_{h} h^{\prime}+c I_{\mu} .
$$


Using these in (4.7), we get

$$
y^{\prime}=a_{0}+a_{1} y-a_{2} y^{2},
$$

where $a_{0}, a_{1}$ and $a_{2}$ are defined in (1.15)-(1.17).

The derivation of the differential equation along backward characteristics,

$$
q^{\prime}=a_{0}-a_{1} q-a_{2} q^{2},
$$

where $q$ is defined in (1.11), is similar, and the proof is complete.

COROLlary 3. A singularity (gradient blowup) forms if and only if

$$
|y| \text { or }|q| \rightarrow \infty \text { iff }\left|u_{x}\right| \text { or }\left|v_{x}\right| \rightarrow \infty,
$$

provided the solution takes values in the compact set $\mathcal{K}$.

Proof. By (1.10) and (1.11),

$$
\begin{aligned}
& y+q=\sqrt{c}(\alpha+\beta), \\
& y-q=\sqrt{c}(\alpha-\beta)-2 I .
\end{aligned}
$$

By (4.3), (1.12) and compactness, $I$ remains finite, and the result follows. $\square$

Because the coefficients $a_{0}, a_{1}$, and $a_{2}$ in Theorem 2 don't include derivative terms $v_{x}, u_{x}, v_{t}$ or $u_{t}$, they are lower order when compared to $y$ and $q$. Using (2.8) and (2.9), these coefficients can be expressed in terms of $v$ and $\bar{x}$ rather than $h$ and $\mu$, as in Corollary 2

In the $p$-system, $p=p(v)$ and $p_{\mu}=0$, so that $a_{0}=a_{1}=0$. In this case, (1.13) and (1.14) become

$$
y^{\prime}=-a_{2} y^{2}, \quad q^{\prime}=-a_{2} q^{2},
$$

which are exactly the ODEs derived in 7,8, for the isentropic homogeneous case.

5. Formation of singularity. We now consider the formation of singularities, which take the form of the blowup of gradients $u_{x}$ and/or $v_{x}$, and correspond to shock formation in conservative systems.

We will study the equations (1.13), (1.14) as a dynamical system, even though this is not a pure system of ODEs. First, consider the ODE

$$
\dot{\xi}=\psi_{ \pm}(\xi)
$$

where $\psi_{ \pm}$is defined by

$$
\psi_{ \pm}(\xi) \equiv a_{0} \pm a_{1} \xi-a_{2} \xi^{2}
$$

with $a_{2}>0$, and the $a_{i}$ are treated as constants. The equilibria, if they exist, are the roots of the quadratic equation

$$
a_{0} \pm a_{1} \xi-a_{2} \xi^{2}=0
$$

and we have $\dot{\xi}>0$ between the roots, and $\dot{\xi}<0$ otherwise. A typical phase line is shown in Figure 5.1 .

Whenever there are real roots, say $\xi_{1} \leq \xi_{2}$, the region $\left\{\xi>\xi_{1}\right\}$ is an invariant region for the ODE. In particular, if $a_{0}>0$, then the roots are of opposite signs and 


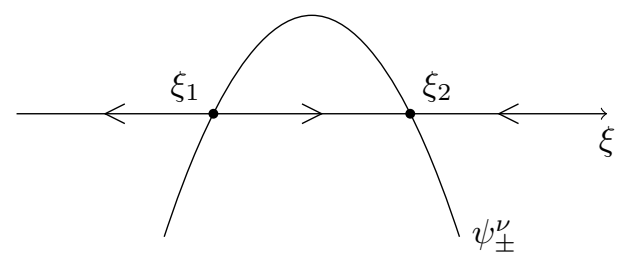

FIG. 5.1. Phase line for $\dot{\xi}=\psi_{ \pm}^{\nu}(\xi)$.

the region $\{\xi>0\}$ is invariant. Moreover, the region $\left\{\xi<\xi_{1}\right\}$ is also invariant, and solutions that originate in this interval have only a finite time of existence: it is this that drives the gowth and blowup of gradients in the full system (4.8), (4.9).

For the $p$-system, we have $p_{\mu}=0$, so $a_{0}=a_{1}=0$ and $I=0$. In this case, $y=$ $\sqrt{c} s_{x}$ and $q=\sqrt{c} r_{x}$ are (multiples of) the gradients of the Riemann invariants. By the above discussion, the regions $\{y>0\},\{q>0\}$ are invariant domains for the system (4.10), as are the regions $\{y<0\},\{q<0\}$. Thus if $y$ or $q$ is negative somewhere, the negative quadratic functions in (4.10) drive blowup of $y$ or $q$ in finite time, as shown by Lax in [7. From our point of view this is a trivial case, for which we have uniform estimates. In general, the forward and backward waves interact nonlinearly with the varying stationary background, and we do not expect uniformity. In fact, interactions can cause waves to change their $R / C$ character, as demonstrated in [14] and the upcoming paper 2. We expect that a complete analysis of the dynamics of (1.13), (1.14) will yield a rich variety of new and unexpected phenomena.

We now prove the breakdown results of Theorem 3 by studying (4.8), (4.9) as a dynamical system. Our aim is to describe sufficient conditions which imply that the gradient blows up in finite time.

Proof of Theorem [3. Fix a constant $0<\nu \ll 1$, and define

$$
\psi_{ \pm}^{\nu}(\xi) \equiv a_{0} \pm a_{1} \xi-(1-\nu) a_{2} \xi^{2},
$$

so that our ODEs (4.8), (4.9) can be written

$$
y^{\prime}=\psi_{+}^{\nu}(y)-\nu a_{2} y^{2} \quad \text { and } \quad q^{\prime}=\psi_{-}^{\nu}(q)-\nu a_{2} q^{2} .
$$

Now let $N=N(\nu)<0$ be a uniform lower bound for the (real) roots of $\psi_{ \pm}^{\nu}$, or $N=0$ if there are no real roots. Then since $a_{2}>0$, we have

$$
\psi_{ \pm}^{\nu}(\xi) \leq 0 \quad \text { for every } \quad \xi \leq N .
$$

Now suppose there is some $x_{0}$ such that the data satisfies

$$
y_{0}=y\left(x_{0}, 0\right)<N .
$$

Then for the forward characteristic emanating from $\left(x_{0}, 0\right)$, we have

$$
y^{\prime} \leq-\nu a_{2} y^{2},
$$

so that the solution satisfies

$$
\frac{1}{y(t)} \geq \frac{1}{y(0)}+\nu \int_{0}^{t} a_{2} d t
$$


where the integral is taken along the forward characteristic. Since $y(0)<0$ and $a_{2}$ is uniformly positive, there is some finite $T_{*}$ such that the right hand side of (5.4) vanishes, and so we obtain

$$
y(t) \rightarrow-\infty \quad \text { as } \quad t \rightarrow T_{*} .
$$

A similar calculation holds for $q$.

Finally, we calculate the lower bound $N$. The roots of $\psi_{ \pm}^{\nu}$ solve the quadratic equations

$$
\psi_{ \pm}^{\nu}(\xi)=a_{0} \pm a_{1} \xi-(1-\nu) a_{2} \xi^{2}=0
$$

and so are

$$
\xi=\frac{ \pm a_{1} \pm \sqrt{\Delta}}{2(1-\nu) a_{2}}, \quad \text { where } \quad \Delta=a_{1}^{2}+4(1-\nu) a_{0} a_{2} .
$$

The minimum of these is clearly

$$
-\frac{\left|a_{1}\right|+\sqrt{\Delta}}{2(1-\nu) a_{2}}
$$

and the lower bound $N$ is obtained by maximizing the ratios $\left|a_{1}\right| / a_{2}$ and $a_{0} / a_{2}$, so we look for upper bounds for $a_{0}$ and $\left|a_{1}\right|$, and a lower bound for $a_{2}$.

From the expressions (1.15)-(1.17) and (1.12), we obtain the bounds provided $c$ and $c_{h}$ are bounded away from zero, while the quantities

$$
h, c, c_{h}, p_{\mu}, p_{\mu h}, p_{\mu h h}, \text { and } p_{\mu \mu h},
$$

remain finite, and recalling (2.5), the bound follows.

Using (2.8) and (2.9), the bounds can also be expressed by the bounds on the derivatives of $p$ (and $c$ ) with respect to $v$ and $\bar{x}$.

When $a_{0}>0$, the equilibria are on opposite sides of the origin, so the stable invariant region includes the values $\xi_{1}<\xi \leq 0$. If the data can be set up in such a way that $y_{0}$ and $q_{0}$ lie in the interval $\left(\xi_{1}, \infty\right)$ for all $x_{0}$, then the corresponding solution would have finite gradients for all time, and these would be nontrivial shockfree solutions. This topic is part of the authors' ongoing research.

6. Generalized $R / C$ structure. The $R / C$ structure at a single contact discontinuity or entropy jump for the compressible Euler equation is fully analyzed in [14. This is an analysis of how the $R / C$ character changes when a wave crosses a jump discontinuity, where the system is a $p$-system on either side of the jump. Using this $R / C$ structure on an entropy jump, a class of time-periodic solutions in compressible Euler equations with polytropic ideal gas has been studied in a series of papers [14, 15, 16. In this section, we show that the $R / C$ characters we have defined for smooth solutions are consistent with those results. Furthermore, by the study of $R / C$ structures in the generalized Euler equations (1.2), (1.3) with smooth and piecewise smooth entropy fields, we expect eventually to see a large class of time-periodic or quasi-periodic solutions as in [14, 15] with both piecewise smooth and smooth entropy profiles. 
We first consider how the $R / C$ character can change at a fixed point in a smooth solution.

Lemma 2. If $\left(\frac{p_{\bar{x}}}{p_{v}}\right)_{v}<0$ (or equivalently $\left(\frac{p_{\mu}}{c}\right)_{h}<0$ ), the backward $R / C$ character can only change from $C$ to $R$ (resp. $R$ to $C$ ), if the solution is forward $C$ (resp. $R$ ); the forward $R / C$ character can only change from $C$ to $R$ (resp. $C$ to $R$ ), if the solution is backward $R$ (resp. C). If $\left(\frac{p_{\bar{x}}}{p_{v}}\right)_{v}>0$ (or $\left(\frac{p_{\mu}}{c}\right)_{h}>0$ ), all the above $R / C$ character changes will only happen in the opposite direction.

Proof. Suppose the backward $R / C$ character changes at $\left(x_{0}, t_{0}\right)$, then $\beta=0$ at $\left(x_{0}, t_{0}\right)$. Then by (1.9), we have

$$
\beta^{\prime}=\frac{c}{2}\left(\frac{p_{\mu}}{c}\right)_{h} \alpha
$$

which has the sign of $\alpha\left(\frac{p_{\mu}}{c}\right)_{h}$. Thus, the backward wave changes from $C(\beta<0)$ to $R$ $(\beta>0)$ at $\left(x_{0}, t_{0}\right)$, if and only if $\alpha$ and $\left(\frac{p_{\mu}}{c}\right)_{h}$ have the same sign. Thus if $\left(\frac{p_{\mu}}{c}\right)_{h}<0$, then $\alpha<0$, and the crossing forward wave is necessarily compressive by Corollary 1 . All other cases follow similarly, and using Corollary 2 for the equivalence of derivative conditions completes the proof. $\square$

In order to compare the $R / C$ structure for smooth solutions to that of a single entropy jump, we recall the relevant argument from [14. Discontinuities in weak solutions are governed by the Rankine-Hugoniot jump conditions, which are

$$
\sigma[v]=-[u], \quad \text { and } \quad \sigma[u]=[p],
$$

plus a third equation for energy conservation. Here $\sigma$ is the speed of the discontinuity, and brackets denote the jump $[f]=f_{R}-f_{L}$ in $f$ across the discontinuity. An entropy jump has zero speed, $\sigma=0$, so that (6.1) reduce to $[u]=0=[p]$, that is

$$
u_{R}=u_{L}, \quad \text { and } \quad p_{R}=p_{L} .
$$

Across the entropy jump, the $R / C$ structure changes as follows, see [14:

Lemma 3. For $\frac{c_{R}}{c_{L}}<1$, the backward $R / C$ character can only change from $R$ to $C$ (resp. $C$ to $R$ ), if the solution is forward $R($ resp. $C$ ); the forward $R / C$ character can only change from $C$ to $R$ (resp. $R$ to $C$ ), if the solution is backward $R$ (resp. $C$ ). For $\frac{c_{R}}{c_{L}}>1$, all the above $R / C$ character changes will only happen in the opposite direction.

Comparing these Lemmas, we see that the condition $\frac{c_{R}}{c_{L}}<1$ for an entropy jump should be consistent with the condition $\left(\frac{p_{\bar{x}}}{p_{v}}\right)_{v}<0$ for smooth solutions. Recalling that the change in pressure at an entropy jump is zero, we can assume $p_{x}=0$, and treat the condition $\frac{c_{R}}{c_{L}}<1$ as $c_{x}<0$. Thus it suffices to show that, if $p_{x}=0$, then

$$
\left(\frac{p_{\bar{x}}}{p_{v}}\right)_{v}<0 \Leftrightarrow c_{x}<0 .
$$

Since $f_{x}=f_{v} v_{x}+f_{\bar{x}}$, if $p_{x}=0$, we have

$$
v_{x}=-\frac{p_{\bar{x}}}{p_{v}}
$$

so that also

$$
c_{x}=c_{v} v_{x}+c_{\bar{x}}=-c_{v} \frac{p_{\bar{x}}}{p_{v}}+c_{\bar{x}}=\frac{-c_{v} p_{\bar{x}}+c_{\bar{x}} p_{v}}{p_{v}} .
$$


On the other hand,

$$
\left(\frac{p_{\bar{x}}}{p_{v}}\right)_{v}=\frac{p_{\bar{x} v} p_{v}-p_{\bar{x}} p_{v v}}{\left(p_{v}\right)^{2}}=-\frac{2 c\left(c_{\bar{x}} p_{v}-p_{\bar{x}} c_{v}\right)}{\left(p_{v}\right)^{2}}
$$

where we recall $p_{v}=-c^{2}$. Comparing these, it follows that

$$
\left(\frac{p_{\bar{x}}}{p_{v}}\right)_{v}=-\frac{2 c}{p_{v}} c_{x}=\frac{2}{c} c_{x}
$$

which proves (6.2). Thus our continuous $R / C$ character is consistent with that of a single entropy jump.

7. Transverse flow of 1D MHD. In this section, we consider the motion of a compressible fluid coupled a magnetic field $H=\left(H_{1}, H_{2}, H_{3}\right)$, which satisfy the Magnetohydrodynamic (MHD) equations in Lagrangian coordinates. In one space dimension, the MHD equations can be written in the non-conservative form

$$
\begin{aligned}
\frac{\partial v}{\partial t}-\frac{\partial u_{1}}{\partial x} & =0, \\
\frac{\partial H_{2}}{\partial t}+\rho H_{2} \frac{\partial u_{1}}{\partial x}-\rho H_{1} \frac{\partial u_{2}}{\partial x} & =0, \\
\frac{\partial H_{3}}{\partial t}+\rho H_{3} \frac{\partial u_{1}}{\partial x}-\rho H_{1} \frac{\partial u_{3}}{\partial x} & =0, \\
\frac{\partial u_{1}}{\partial t}+\frac{\partial}{\partial x}\left(p+\frac{1}{2} \mu_{0}\left(H_{2}^{2}+H_{3}^{2}\right)\right) & =0, \\
\frac{\partial u_{2}}{\partial t}-\mu_{0} H_{1} \frac{\partial H_{2}}{\partial x} & =0, \\
\frac{\partial u_{3}}{\partial t}-\mu_{0} H_{1} \frac{\partial H_{3}}{\partial x} & =0, \\
\frac{\partial S}{\partial t} & =0,
\end{aligned}
$$

where $\rho$ is the density, $v=\rho^{-1}$ is the specific volume, $\left(u_{1}, u_{2}, u_{3}\right)$ is the velocity field, $S$ is the entropy, and $p=p(v, S)$ is the pressure. The permeability $\mu_{0}$ is a positive constant, and $H_{1}$ is constant in the one-dimesional model, c.f. 9, 12].

The mathematical structures of (7.1)-(7.7) are totally different when $H_{1}$ is zero or a nonzero constant. Here we briefly consider the easier case $H_{1}=0$, which physically means that the magnetic field is transverse to the direction of motion. In this case, (7.1) - (17.4) and (7.7) form a closed system [9]:

$$
\begin{aligned}
\frac{\partial v}{\partial t}-\frac{\partial u_{1}}{\partial x} & =0, \\
\frac{\partial u_{1}}{\partial t}+\frac{\partial \tilde{p}}{\partial x} & =0 \\
\frac{\partial \tilde{H}_{2}}{\partial t} & =0 \\
\frac{\partial \tilde{H}_{3}}{\partial t} & =0 \\
\frac{\partial S}{\partial t} & =0
\end{aligned}
$$


where

$$
\tilde{H}_{2}=v H_{2}, \quad \tilde{H}_{3}=v H_{3}, \quad \tilde{p}=p+\frac{1}{2} \mu_{0} \frac{\tilde{H}_{2}^{2}+\tilde{H}_{3}^{2}}{v^{2}} .
$$

Thus in the special case of transverse flow, the one-dimensional MHD equations fit into the framework of (1.2), (1.3), and our previous results apply directly.

We analyze this further for a polytropic ideal ( $\gamma$-law) gas, so that the pressure is given by

$$
p=K e^{S / c_{v}} v^{-\gamma},
$$

with adiabatic gas constant $\gamma>1$, and $K, c_{v}$ are positive constants. Then we get

$$
\begin{aligned}
\tilde{p} & =K e^{S / c_{v}} v^{-\gamma}+\frac{1}{2} \mu_{0} \frac{\tilde{H}_{2}^{2}+\tilde{H}_{3}^{2}}{v^{2}} \\
& =A_{1} v^{-\gamma}+A_{2} v^{-2}
\end{aligned}
$$

where we have set

$$
A_{1}(x) \equiv K e^{S(x) / c_{v}} \quad \text { and } \quad A_{2}(x) \equiv \frac{1}{2} \mu_{0}\left(\tilde{H}_{2}^{2}(x)+\tilde{H}_{3}^{2}(x)\right) .
$$

In order to further simplify our calculation, we now assume $\gamma=2$, and set

$$
B(x) \equiv A_{1}(x)+A_{2}(x), \quad \text { so that } \quad \tilde{p}(v, x)=B(x) v^{-2} .
$$

We then calculate

$$
c(v, x)=\sqrt{-p_{v}}=\sqrt{2} \sqrt{B(x)} v^{-3 / 2},
$$

and, from (1.7),

$$
h(v, x)=\int_{v}^{\infty} c d v=2 \sqrt{2} \sqrt{B(x)} v^{-1 / 2} .
$$

Now solving for $v$ and recalling $\mu=x$, we obtain

$$
v(h, \mu)=8 B(\mu) h^{-2},
$$

and so also

$$
p(h, \mu)=\frac{h^{4}}{64 B(\mu)} \quad \text { and } \quad c(h, \mu)=\frac{h^{3}}{16 B(\mu)} .
$$

Note that

$$
\frac{\partial p}{\partial h}(h, \mu)=c(h, \mu) \quad \text { and } \quad \frac{\partial v}{\partial h}(h, \mu)=\frac{-1}{c(h, \mu)},
$$

as intended by the choice of $h$, see [17]. Next, we calculate

$$
\frac{p_{\mu}}{c}=-\frac{h}{4} \frac{\dot{B}(\mu)}{B(\mu)},
$$


where $\dot{B} \equiv \frac{d B}{d \mu}$, so that

$$
\alpha=u_{x}+h_{x}-\frac{h}{4} \frac{\dot{B}(x)}{B(x)} \quad \text { and } \quad \beta=u_{x}-h_{x}+\frac{h}{4} \frac{\dot{B}(x)}{B(x)} ;
$$

here $\alpha$ and $\beta$ are evaluated at the point $(x, t)$, and $\mu=x$. From (1.12), we calculate

$$
I(h, \mu)=\frac{-1}{80} \frac{\dot{B}(\mu)}{B(\mu)^{3 / 2}} h^{5 / 2},
$$

which also leads to

$$
\frac{p_{\mu}}{\sqrt{c}}-I=\frac{-1}{20} \frac{\dot{B}(\mu)}{B(\mu)^{3 / 2}} h^{5 / 2},
$$

and thus, by (1.10), (1.11),

$$
y=\frac{h^{3 / 2}}{4 \sqrt{B}}\left(u_{x}+h_{x}-\frac{h}{5} \frac{\dot{B}}{B}\right), \quad \text { and } \quad q=\frac{h^{3 / 2}}{4 \sqrt{B}}\left(u_{x}-h_{x}+\frac{h}{5} \frac{\dot{B}}{B}\right) .
$$

Finally, using (1.15)-1.17), we calculate the coefficients

$$
a_{2}=\frac{3}{8} \frac{h^{1 / 2}}{\sqrt{B}}, \quad \text { and } \quad a_{1}=\frac{1}{40} \frac{\dot{B}}{B^{2}} h^{3}=-2 \frac{h^{1 / 2}}{\sqrt{B}} I,
$$

where we have used (7.9), and, after simplification,

$$
\begin{aligned}
a_{0} & =h^{11 / 2}\left[\frac{1}{2^{8} 5} \frac{1}{B}\left(\frac{\dot{B}}{B^{3 / 2}}\right)+\frac{\dot{B}^{2}}{B^{7 / 2}}\left(\frac{1}{2^{11}}-\frac{1}{2^{10} 5}-\frac{3}{2^{11} 5^{2}}\right)\right] \\
& =\frac{h^{11 / 2}}{2^{8} 5}\left[\frac{\ddot{B}}{B^{5 / 2}}-\frac{6}{5} \frac{\dot{B}^{2}}{B^{7 / 2}}\right]=-6 \frac{h^{1 / 2}}{\sqrt{B}} G(\mu) I^{2},
\end{aligned}
$$

where we have set

$$
G(\mu) \equiv 1-\frac{5}{6} \frac{B(\mu) \ddot{B}(\mu)}{\dot{B}(\mu)^{2}}
$$

wherever $\dot{B}(\mu) \neq 0$.

We use these in (5.1) to calculate the quadratic

$$
\psi_{ \pm}(\xi)=-\frac{h^{1 / 2}}{\sqrt{B}}\left(6 G I^{2} \pm 2 I \xi+\frac{3}{8} \xi^{2}\right)
$$

and the corresponding dynamical system can be analyzed as above. It is clear that the growth of the quantities $y$ and/or $q$ depends critically on their size relative to $I$.

8. Euler flow in a variable area duct. Finally, we consider the compressible Euler flow through a duct of varying cross section $a(\tilde{x})$. In (spatial) Eulerian coordinates $(\tilde{x}, \tilde{t})$, this flow satisfies:

$$
\begin{aligned}
a_{\tilde{t}} & =0, \\
(a \rho)_{\tilde{t}}+(a \rho u)_{\tilde{x}} & =0, \\
(a \rho u)_{\tilde{t}}+\left(a \rho u^{2}\right)_{\tilde{x}}+a p_{\tilde{x}} & =0, \\
(a \rho E)_{\tilde{t}}+(a \rho E u+a p u)_{\tilde{x}}=0, &
\end{aligned}
$$


where $E$ is the energy, $\rho$ is the density, $u$ is the velocity, $p$ is the pressure and $a=a(\tilde{x})$ is the diameter of the duct at position $\tilde{x}, 3,4,11$. For smooth solutions, the energy equation (8.4) is equivalent to

$$
S_{\tilde{t}}+u S_{\tilde{x}}=0,
$$

where $S$ is the entropy [3]. By introducing Lagrangian coordinates $(x, t)$, which satisfy

$$
\begin{aligned}
d x & =a \rho d \tilde{x}-a \rho u d \tilde{t}, \\
d t & =d \tilde{t},
\end{aligned}
$$

it is easy to check the smooth solutions of $(8.1)-(8.3)$ and (8.5) satisfy

$$
\begin{aligned}
\hat{v}_{t}-u_{x} & =0, \\
u_{t}+a p_{x} & =0, \\
S_{t} & =0,
\end{aligned}
$$

where $\hat{v}$ is the specific volume per unit cross-sectional area,

$$
\hat{v}=\frac{1}{a \rho} .
$$

By by (8.2), the right hand side of (8.6) is an exact differential, so there is no difficulty defining $x$. By (8.6), (8.7), $a(x, t)$ satisfies the identities

$$
a_{t}=u \dot{a}, \quad a_{x}=\hat{v} \dot{a}, \quad(\dot{a})_{t}=u \ddot{a}, \quad \text { and } \quad(\dot{a})_{x}=\hat{v} \ddot{a},
$$

where we denote

$$
\dot{a}=\frac{d a(\tilde{x})}{d \tilde{x}}, \quad \text { and } \quad \ddot{a}=\frac{d^{2} a(\tilde{x})}{d \tilde{x}^{2}},
$$

these describing the changing shape of the duct in spatial coordinates.

We again assume we have an ideal polytropic gas, so that

$$
p=K e^{S / c_{v}} v^{-\gamma}=K e^{S / c_{v}}(a \hat{v})^{-\gamma} .
$$

with adiabatic gas constant $\gamma>1$, c.f. [3]. The (Lagrangian) sound speed is

$$
c=\sqrt{-a p_{\hat{v}}}=\sqrt{K \gamma} a^{-\frac{\gamma-1}{2}} \hat{v}^{-\frac{\gamma+1}{2}} e^{\frac{S}{2 c_{v}}} .
$$

Following [14, we make the change of variables

$$
m=e^{S / 2 c_{v}} \quad \text { and } \quad z=\int_{\hat{v}}^{\infty} \frac{c}{a^{-\frac{\gamma-1}{2}} m} d \hat{v}=\frac{2 \sqrt{K \gamma}}{\gamma-1} \hat{v}^{-\frac{\gamma-1}{2}} .
$$

It follows that

$$
\begin{aligned}
& \hat{v}=K_{v} z^{-\frac{2}{\gamma-1}}, \\
& p=K_{p} a^{-\gamma} m^{2} z^{\frac{2 \gamma}{\gamma-1}}, \quad \text { and } \\
& c=c(z, m)=K_{c} a^{-\frac{\gamma-1}{2}} m z^{\frac{\gamma+1}{\gamma-1}},
\end{aligned}
$$

where $K_{v}, K_{p}$ and $K_{c}$ are appropriate positive constants, see [14]. 
For $C^{1}$ solutions, the Lagrangian equations (8.8)-(8.10) are equivalent to

$$
\begin{aligned}
z_{t}+\frac{c}{a^{-\frac{\gamma-1}{2}} m} u_{x} & =0, \\
u_{t}+m c a^{-\frac{\gamma-1}{2}} z_{x}+2 \frac{a p}{m} m_{x}-\gamma p a_{x} & =0, \\
m_{t} & =0 .
\end{aligned}
$$

We define

$$
\begin{aligned}
& \alpha=u_{x}+a^{-\frac{\gamma-1}{2}} m z_{x}+\frac{\gamma-1}{\gamma} a^{-\frac{\gamma-1}{2}} m_{x} z \quad \text { and } \\
& \beta=u_{x}-a^{-\frac{\gamma-1}{2}} m z_{x}-\frac{\gamma-1}{\gamma} a^{-\frac{\gamma-1}{2}} m_{x} z,
\end{aligned}
$$

which are the gradient variables used for the polytropic ideal Euler flow in 1], adjusted to account for $a(\tilde{x})$. By similar calculations as in the proof of Theorem 1 or in 1], we obtain

$$
\begin{aligned}
& \alpha^{\prime}=k_{1}\left(k_{2}(3 \alpha+\beta)+\left(\alpha \beta-\alpha^{2}\right)\right)+k_{3}(\alpha-\beta)+A(x, t), \\
& \beta^{\prime}=-k_{1}\left(k_{2}(3 \beta+\alpha)+\left(\alpha \beta-\beta^{2}\right)\right)+k_{3}(\beta-\alpha)-A(x, t),
\end{aligned}
$$

where

$$
A(x, t)=\frac{(\gamma-1)^{3}}{8 K_{c}} m^{2} z^{\frac{2 \gamma-4}{\gamma-1}} a^{-\gamma-1}\left[a \ddot{a}-\gamma \dot{a}^{2}\right]+\frac{(\gamma-1)^{2}}{2 \gamma} m m_{x} z^{2} a^{-\gamma} \dot{a},
$$

and the coefficients are

$$
\begin{aligned}
& k_{1}=\frac{\gamma+1}{2(\gamma-1)} K_{c} z^{\frac{2}{\gamma-1}}, \\
& k_{2}=\frac{\gamma-1}{\gamma \gamma+1} m_{x} z a^{-\frac{\gamma-1}{2}}, \quad \text { and } \\
& k_{3}=\frac{3(\gamma-1)^{3}}{8} m z a^{-\frac{\gamma+1}{2}} \dot{a}-\frac{\gamma-1}{4} u a^{-1} \dot{a} .
\end{aligned}
$$

By (8.13) -8.15 ,

$$
z^{\prime}=z_{t}+c z_{x}=-K_{c} z^{\frac{\gamma+1}{\gamma-1}}\left(\beta+\frac{\gamma-1}{\gamma} m_{x} z a^{-\frac{\gamma-1}{2}}\right),
$$

so that

$$
\beta=-\frac{z^{\prime}}{K_{c} z^{\frac{\gamma+1}{\gamma-1}}}-\frac{\gamma-1}{\gamma} m_{x} z a^{-\frac{\gamma-1}{2}} .
$$

We plug (8.18) into (8.16), move terms including $z^{\prime}$ to the left hand side, and multiply by $z^{\frac{\gamma+1}{2(\gamma-1)}}$ on both sides, to obtain

$$
\begin{aligned}
\left(z^{\frac{\gamma+1}{2(\gamma-1)}} \alpha\right)^{\prime} & +\frac{m_{x}}{2 \gamma} a^{-\frac{\gamma-1}{2}} z^{\frac{\gamma+1}{2(\gamma-1)}} z^{\prime}-\frac{1}{K_{c}} k_{3} z^{-\frac{\gamma+1}{2(\gamma-1)}} z^{\prime} \\
= & k_{1}\left(k_{2}\left(3 \alpha-\frac{\gamma-1}{\gamma} m_{x} z a^{-\frac{\gamma-1}{2}}\right)+\left(-\frac{\gamma-1}{\gamma} m_{x} z a^{-\frac{\gamma-1}{2}} \alpha-\alpha^{2}\right)\right) z^{\frac{\gamma+1}{2(\gamma-1)}} \\
& +k_{3}\left(\alpha+\frac{\gamma-1}{\gamma} m_{x} z a^{-\frac{\gamma-1}{2}}\right) z^{\frac{\gamma+1}{2(\gamma-1)}}+A(x, t) z^{\frac{\gamma+1}{2(\gamma-1)}}
\end{aligned}
$$


Furthermore, by (8.13)-(8.15), we have

$$
\begin{aligned}
u^{\prime} & =u_{t}+c u_{x}=c \beta+\gamma p a_{x} \\
& =-m a^{-\frac{\gamma-1}{2}} z^{\prime}-\frac{\gamma-1}{\gamma} c z m_{x} a^{-\frac{\gamma-1}{2}}+\gamma p a_{x} .
\end{aligned}
$$

By (8.20) and following the proof of Theorem 2, (8.19) can again be transformed into

$$
y^{\prime}=\psi_{+}(y)=a_{0}+a_{1} y-a_{2} y^{2},
$$

where $y$ is a gradient variable, and $a_{0}, a_{1}$ are functions of $m, m_{x}, m_{x x}, z, a, \dot{a}, \ddot{a}$, and $u$, and

$$
a_{2}=\frac{\gamma+1}{2(\gamma-1)} K_{c} z^{\frac{\gamma+1}{2(\gamma-1)}-1}>0 .
$$

Similar considerations for backward waves yield a similar equation for the gradient variable $q$, namely

$$
q^{\prime}=\psi_{-}(q)=a_{0}-a_{1} q-a_{2} q^{2} .
$$

A blowup result similar to Theorem 3 can again be obtained for this system. We omit the details since the calculations are tedious but similar to our previous calculation, and $a_{0}$ and $a_{1}$ are more complicated expressions. Note in particular that they depend on $u$ as well as $z$, which is a new feature of this case.

\section{REFERENCES}

[1] G. Chen, Formation of singularity and smooth wave propagation for the non-isentropic compressible Euler equations, submitted, available at http://www.math.ntnu.no/conservation/2010/020.html

[2] G. Chen, R. Young, Global shock free solutions and shock formation for the compressible Euler equations with monotonic entropy, in preparation.

[3] R. Courant and K. O. Friedrichs, Supersonic Flow and Shock Waves, Wiley-Interscience, New York, 1948.

[4] C. M. Dafermos, Hyperbolic Conservations laws in Continuum Physics, Springer-Verlag, Heidelberg, 2000.

[5] F. John, Formation of Singularities in One-Dimensional Nonlinear Wave Propagation, Comm. Pure Appl. Math. 27(1974) 377-405.

[6] F. John, Formation of singularities in elastic waves, Lecture Notes in Physics, 195(1984) 194-210.

[7] P. D. Lax, Development of singularities of solutions of nonlinear hyperbolic partial differential equations, J. Math. Physics 5:5(1964), 611-614.

[8] P. D. Lax, Hyperbolic systems of conservation laws and the mathematical theory of shock waves, Conf. Board Math. Sci. 11, SIAM, 1973.

[9] T. Li and T. Qin, Physics and partial differential equations, Volume 1 (in Chinese), Higher Education press, Beijing, 1997.

[10] T.-P. Liu, The development of singularities in the nonlinear waves for quasi-linear hyperbolic partial differential equations, Jour. Diff. Equations, 33, (1979) 92-111.

[11] J. Hong and B. Temple, A bound on the total variation of the conserved quantities for solutions of a general resonant nonlinear balance law, SIAM J. Appl. Math. 64:3, 819-857.

[12] M. A. Rammaha, Formation of singularities in compressible fluids in two-space dimensions, Proc. Amer. Math. Soc. 107:3, (1989) 705-714.

[13] T. Sideris, Formation of singularities in three-dimensional compressible fluids, Commun. Math. Phys., 101, (1985) 475-485.

[14] B. Temple and R. Young, A Paradigm for Time-Periodic Sound Wave Propagation in the Compressible Euler Equations, Methods Appl. Anal., 16:3(2009), 341-364. 
[15] B. Temple and R. Young, Time-Periodic Linearized Solutions of The Compressible Euler Equations and a Problem of Small Divisors, to appear in SIAM Jour. of Math. Anal..

[16] B. Temple and R. Young, A Liapunov-Schmidt Reduction for Time-Periodic Solutions of the Compressible Euler Equations, submitted.

[17] R. Young, Global wave interactions in isentropic gas dynamics, to appear.

[18] R. Young, Convergence of characteristics and shock formation, in preparation. 